\title{
Using the interface Peclet number to select the maximum simulation interface width in phase-field solidification modelling
}

\author{
Y. Xie ${ }^{\text {a }}$, H.B. Dong ${ }^{\text {a,* }}$, J.A. Dantzig ${ }^{\mathrm{b}, \mathrm{c}}$ \\ a Department of Engineering, University of Leicester, Leicester LE1 7RH, UK \\ ${ }^{\mathrm{b}}$ Laboratory for the Simulation of Materials, EPFL, Lausanne 1015, Switzerland \\ ${ }^{\mathrm{c}}$ Department of Mechanical Science and Engineering, University of Illinois, Urbana, IL 61801, USA
}

\section{A R T I C L E I N F O}

Article history:

Received 29 August 2012

Received in revised form 14 November 2012

Accepted 17 December 2012

Available online 30 January 2013

\section{Keywords:}

Dendritic solidification

Phase-field modelling

Peclet number

Steels

\begin{abstract}
A B S T R A C T
This study investigates the use of interface Peclet number $P=w /\left(D_{l} / V_{t i p}\right)$, to determine the interface width $(w)$ used in phase-field simulations, where $D_{l}$ is the liquid diffusivity and $V_{t i p}$ is the tip velocity. The maximum simulation interface width $\left(w_{\max }\right)$ under varied growth conditions was analysed via convergence analysis and it was found that there is a limit of $P$ for the maximum interface width at various velocities. Converged results can be obtained only when $w<w_{\max }=0.075 D_{l} / V_{\max }$ where $V_{\max }$ is the maximum growth velocity during transient solidification. The effect of the inclusion of finite solid diffusivity on the $P$ limit in selecting $w_{\max }$ was analysed, and little influence was observed.
\end{abstract}

(C) 2013 Elsevier B.V. All rights reserved.

\section{Introduction}

Dendrites are one of the basic microstructural patterns in solidified materials $[1,2]$. Due to the complex evolution of the solid/liquid (S/L) interface morphology during dendritic growth, the phase-field (PF) method is now commonly used to simulate dendritic growth because it circumvents the direct tracking the S/L interface. The PF method introduces a new variable $\phi$ to distinguish different fields [3]: $\phi=+1$ in solid, $\phi=-1$ in liquid, and intermediate values correspond to the $\mathrm{S}-\mathrm{L}$ interface. Thus, the $\mathrm{S} / \mathrm{L}$ interface is treated as a diffuse field with a non-zero width $(w)$. Various PF solidification models have been developed for binary [4-12], ternary [13-19] and multi-phase [20-26] alloys.

Quantitative predictions using real physical length and time scales remain a major challenge, however, because both the diffuse interface width $(w)$ and the characteristic interface kinetic time scale $(\tau)$ are nano-scale; $w$ is a few angstroms and $\tau$ is roughly the ratio of $w$ and the thermal velocity of atoms in the liquid $[27,28]$. In contrast, diffusive solute transport in bulk phases occurs on microscopic length and time scales, which are several orders of magnitude larger than $w$ and $\tau$. Spanning from nano-scale to micro-scale length/time scale in phase-field simulations is computationally impractical.

To improve the calculation efficiency, a larger interface width, i.e. $w>w^{*}$, is needed in PF simulations and the key is to maintain

\footnotetext{
* Corresponding author. Tel.: +44 116 2522528; fax: +44 1162522525

E-mail address: hd38@le.ac.uk (H.B. Dong).
}

the Gibbs-Thomson relation and the mass conservation at this expanded interface. Karma and Rappel [29-31] presented a thininterface asymptotic analysis for pure systems with equal diffusivities in both the solid and liquid phases to define the PF parameters that ensure the maintenance of the Gibbs-Thomson relation and the mass conservation. In alloys, where the ratio of diffusivities is usually very small, the finite width of the interface traps solute artificially, leading to erroneous results. Karma [8] introduced an anti-trapping current to suppress the artificial solute trapping, which was also evaluated by a similar thin-interface analysis. Later, Echebarria et al. [9] extended this to the directional solidification. The thin-interface analysis corrects for dependencies within the solution that is linear in the expansion parameter $w / d_{0}$, where $d_{0}$ is the solutal capillary length scale, defined as $d_{0}=-\frac{\Gamma_{s l}}{m_{e} C_{l}^{0}\left(1-k_{e}\right)}$ where $\Gamma_{s l}$ is the Gibbs-Thomson coefficient, $m_{e}$ is the liquidus slope, $k_{e}$ is the equilibrium partition coefficient and $C_{l}^{0}$ is the liquid composition at isothermal temperature. The magnitude of $d_{0}$ is on the order of $10^{-8} \mathrm{~m}$. Second and higher order dependencies remain, which implies there is an upper limit of $w$, beyond which the simulation results will not converge to the correct result.

The interface width $w$ is selected using a "capillary selection criterion" (the value of $w / d_{0}$ ). Various values of $w / d_{0}$ have been used in the literature to select the interface width in phase-field simulations, as listed in Table 1 . In the various cases, the validation of the selected $w / d_{0}$ value was made by comparing the calculated results with the predictions of Gibbs-Thomson relation [8] or the MullinsSekerka instability calculation [9], or by performing a convergence study [9]. It should be noted that the values of $w / d_{0}$ are much 
Table 1

Values of $w / d_{0}$ used in selecting interface width in phase-field simulations.

\begin{tabular}{lll}
\hline$w / d_{0}$ & Methods used to validate the selection of $w / d_{0}$ values & Refs. \\
\hline 1.83 & Compare with Gibbs-Thomson relation & {$[8]$} \\
3.6 & Compare with Gibbs-Thomson relation & {$[8]$} \\
9.025 & Compare with Mullins-Sekerka instability & {$[9]$} \\
38.0 & Convergence analysis & {$[9]$} \\
54.0 & Convergence analysis & {$[9]$} \\
72.1 & Convergence analysis & {$[9]$} \\
\hline
\end{tabular}

larger than one, which deviates from the expected range for an expansion parameter. Also, $w / d_{0}$ spans a large range, from 1.83 [8] to 72.2 [9], as listed in the table. In particular, no limit to the maximum interface width $\left(w_{\max } / d_{0}\right)$ is implied by the capillary criterion.

Dendrites do not grow under steady-state condition but under transient growth condition in practice. Under conventional casting conditions, the dendrite growth velocity is highest near the chilling wall, and decreases with distance from it [32,33]. The dendrite growth velocity varies significantly with position in a weld pool [34]. To select a proper interface width to simulate the transient dendrite growth, the $w / d_{0}$ criterion becomes insufficient because this criterion is not related to the solidification condition under a certain alloy system.

Echebarria et al. [9] suggested the possibility to use the interface Peclet number $P=w /\left(D_{l} / V_{\text {tip }}\right)$, where $D_{l}$ is the liquid diffusivity and $V_{\text {tip }}$ is the tip velocity, as a small expansion parameter, to substitute the role of $w / d_{0}$, in the thin-interface analysis. The $P$ criterion can correlate the interface width with growth velocity $\left(V_{t i p}\right)$. However, this option was not pursued in their derivation because of the interface stretching and surface diffusion terms appearing at second order and third order in an expansion in the interface Peclet number. In this study we examined the limit of the interface Peclet number to the maximum interface width through numerical simulations instead. It should be pointed out that using the interface Peclet number to select an interface width prior to the simulation is limited because $V_{t i p}$ is not known a priori. In such a case, the interface Peclet number criterion can be used to evaluate the appropriateness of the selected interface width. As an extended application for modelling solidification in Fe-C alloys, the effect of solid diffusivity on selecting $P$ limit corresponding to the maximum interface width was also investigated.

\section{Methods description}

\subsection{Thin-interface phase-field solidification model}

In this section, the PF model is summarized, and we refer the interested reader to the original article for further details $[9,10]$. The phase field variable is $\phi ; \phi=+1$ in solid, $\phi=-1$ in liquid, and it changes from +1 to -1 across $S / L$ interface. For a dilute binary alloy under isothermal solidification, the PF governing equations consist of solute diffusion equation and phase field equation. The solute diffusion equation without convection is based on Fick's law, expressed as:

$\frac{\partial C}{\partial t}=\nabla \cdot\left[\tilde{q}(\phi) D_{l} \nabla C\right]$

where $C$ is the solute concentration, $t$ is time, and $\nabla$ is the gradient operator. $\tilde{q}(\phi) D_{l}$ is the solute diffusivity for the entire solidification domain; $\tilde{q}(+1)=D_{s} / D_{l}$ in solid, $\tilde{q}(-1)=1$ in liquid. We next define the dimensionless variable $u$ as:

$u=\ln \frac{C}{\left[\left(C_{l}^{0}+C_{s}^{0}\right) / 2+h(\phi)\left(C_{s}^{0}-C_{l}^{0}\right) / 2\right]}$ where $C_{l}^{0}, C_{s}^{0}$ is the equilibrium solute concentration in liquid and solid at the isothermal solidification temperature, and it has $C_{s}^{0}=k_{e} C_{l}^{0}$. The interpolated equilibrium composition $C^{0}$ across the entire solidification domain is $\left(C_{l}^{0}+C_{s}^{0}\right) / 2+h(\phi)\left(C_{s}^{0}-C_{l}^{0}\right) / 2$ with the interpolation function $h(\phi)$ defined below. Eq. (2) shows that $u$ is a measure of the deviation of solute concentration $C$ from the equilibrium concentration $C^{0}$. Then, Eq. (1) becomes:

$$
\frac{\partial C}{\partial t}=\nabla \cdot\left[\tilde{q}(\phi) D_{l} e^{u} \frac{C_{l}^{0}\left[1+k_{e}-\left(1-k_{e}\right) h(\phi)\right]}{2} \nabla u\right]
$$

To reduce the artificial solute trapping, an anti-trapping term was added and Eq. (3) becomes:

$\frac{\partial C}{\partial t}=\nabla \cdot\left[\tilde{q}(\phi) D_{l} e^{u} \frac{C_{l}^{0}\left[1+k_{e}-\left(1-k_{e}\right) h(\phi)\right]}{2} \nabla u\right]-\nabla \cdot j_{a t}$

with the anti-trapping current term expressed as:

$j_{a t}=-a(\phi) w\left(1-k_{e}\right) C_{l}^{0} e^{u} \frac{\partial \phi}{\partial t} \frac{\nabla \phi}{|\nabla \phi|}$

with $a(\phi)$ described further below. The anti-trapping current is proportional to the interface width $w$ and $\partial \phi / \partial t$ that implies the velocity of every point, and is related to equilibrium partition coefficient $k_{e}$. See [9] for further details.

The phase-field evolution equation is given as:

$\tau \frac{\partial \phi}{\partial t}=w^{2} \nabla^{2} \phi+\left(\phi-\phi^{3}\right)-\frac{\lambda}{\left(1-k_{e}\right)}\left(1-\phi^{2}\right)^{2}\left(e^{u}-1\right)$

where $\tau$ is the characteristic kinetic time constant and $\lambda$ is a dimensionless parameter that relates the phase-field length scale $(w)$ to the physical length scale $\left(d_{0}\right)$. To correctly describe the S-L interfacial energy, $\lambda=a_{1} w / d_{0}$ is established, where $a_{1}$ is a constant with the value dependent on the definition of the interpolation function $h(\phi)$ in Eqs. (3) and (4).

The thin-interface limit analysis is performed to define the interface width $w$ and the magnitude of the anti-trapping term $a(\phi)$ in the governing equations to recover the Gibbs-Thomson relation and reduce the artificial solute trapping by assuming zero solid diffusivity. In [9], $\tilde{q}(\phi)$ is expressed as:

$\tilde{q}(\phi)=\frac{(1-\phi)}{1+k_{e}-\left(1-k_{e}\right) \phi}$

If we choose $h(\phi)=\phi$, then $w$ and $a(\phi)$ satisfy:

$\beta=a_{1}\left[1-a_{2} \frac{\lambda w^{2}}{\tau D_{l}}\right]$

$a(\phi)=\frac{1}{2 \sqrt{2}}$

where $\beta$ is the kinetic attachment coefficient, $a_{1}=0.8839$ and $a_{2}=0.6267$

Anisotropy for the cubic symmetry crystal is considered in simulations. The S/L interfacial energy is $\gamma_{s l}=\gamma_{s l}^{0} a_{n}$ with the anisotropy term, $a_{n}$, expressed as:

$a_{n}=1-3 \varepsilon_{4}+4 \varepsilon_{4} \times \frac{n_{x}^{4}+n_{y}^{4}+n_{z}^{4}}{\left(n_{x}^{2}+n_{y}^{2}+n_{z}^{2}\right)^{2}}$

where $\varepsilon_{4}$ is the strength of anisotropy. $n_{x}, n_{y}$ and $n_{z}$ are the Cartesian components of the crystalline direction in the reference frame. The anisotropy is incorporated into the phase-field model by defining $w=w_{0} a_{n}$ and $\tau=\tau_{0} a_{n}^{2}[31]$. 


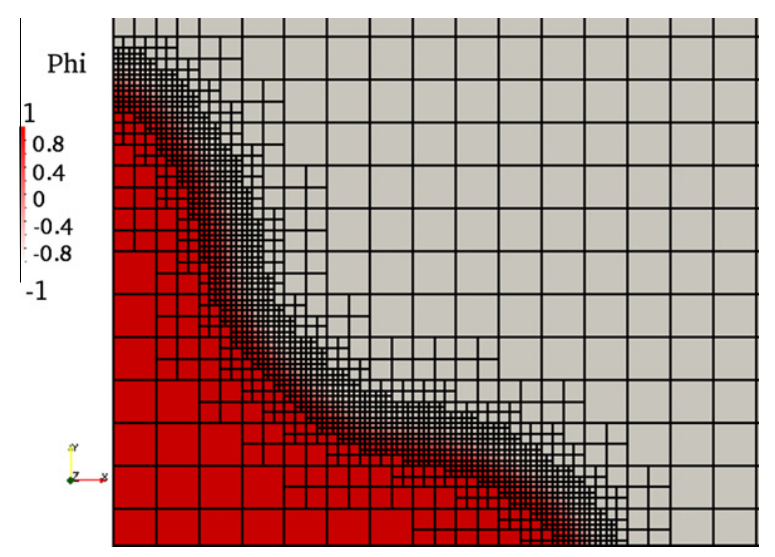

Fig. 1. Adaptive meshes in the in the growing dendrite. Red region represent the solid, grey region the liquid, and otherwise the interface. (For interpretation of the references to colour in this figure legend, the reader is referred to the web version of this article.)

\subsection{Numerical implementation using adaptive meshing algorithm}

A developed code for three-dimensional (3D) PF simulations was used in this study, in which finite element method was used to solve the partial differential equations, i.e. Eqs. (4) and (6). However, to save calculation time and to compare with other simulations in the literature, we preferred two-dimensional (2D) simulations. The transition from the $3 \mathrm{D}$ to $2 \mathrm{D}$ due to the refinement in one direction were observed [1], and therefore thin samples with $L_{x}=L_{y} \gg L_{z}$ were used to realize 2D simulations. $L_{x}$ and $L_{y}$ were chosen to be long enough for the dendrite to grow freely to steady state. Simulations started with an initial seed with radius $r_{0}=22 d_{0}$ and thickness $h=25 d_{0}$ growing in a supersaturated melt with initial concentration $C_{\infty}$. The supersaturation, i.e. $\Omega=\left(C_{l}^{0}-C_{\infty}\right) /\left[C_{l}^{0}\left(1-k_{e}\right)\right]$, varied from 0.45 to 0.60 . The equilibrium partition coefficient of the alloy was 0.15 , i.e. $k_{e}=0.15$, and four-fold anisotropy coefficient $\varepsilon_{4}=0.02$ was used. In the calculations, time step was $\Delta t=0.01 \tau$, where $\tau=a_{1} a_{2} \lambda w^{2} / D_{l}$ with vanishing kinetics according to Eq. (8).

Because the variables, $\phi$ and $C$, changed rapidly only in a narrow region around the interface, the field equations were solved on an adaptive grid to improve the calculation efficiency. The refinement criterion in the simulations is based on a local error estimator that is sensitive to variations in the gradients of both field variables. A detailed description of the adaptive meshing technique and the validation of the error estimator can be found in Refs. $[35,36]$. In the simulations, the maximum grid spacing $(\Delta x)_{\max }$ equated $h$ and the minimum grid spacing $(\Delta x)_{\min }$ was in the range of $(0.4 /$ 1) $w$ to assure numerical convergence.

Fig. 1 provides an example of the adaptive meshing setting in the numerical tests. $\Omega=0.55$ and $w=5 d_{0}$ in this case. In the figure, the maximum grid spacing $(\Delta x)_{\max }=5 w$, the finest grid spacing $(\Delta x)_{\min }=0.625 w$, and the refinement level is 3 . As shown in Fig. 1, the field of the finest grids is about $15 w$ in width, which is not a broad region. A comparison was made between the results using adaptive grids and those using fixed grids in which $\Delta x=(\Delta x)_{\min }=0.625 w$. At time $t=400 \tau$, the tip radius obtained using adaptive grids is $21 d_{0}$, within $2 \%$ of $R_{\text {tip }}=20.7 d_{0}$ obtained using fixed grids, indicating that the adaptive meshing algorithm in simulations could lead to creditable simulation results even when the finest grids are only in a narrow region. The narrow region of the finest grids in this case was due to the use of high $\Omega$ in the simulation which led to the high growth velocity meaning the concentration varied in a narrow region based on $D_{l} / V_{\text {tip }}$.

Steady-state tip radius and tip velocity were computed for the various cases. Fig. 2 shows the typical evolution of the tip radius and tip velocity with time. In the example case, the supersaturation is 0.55 and the interface width is $5 d_{0}$. As shown in Fig. 2, the tip radius increased with time while the tip velocity decreased with time until the steady state was achieved. The selection constant $\sigma^{*}=\sqrt{d_{0} D_{l} /\left(R_{\text {tip }}^{2} V_{\text {tip }}\right)}$ was calculated from the values of $R_{\text {tip }}$
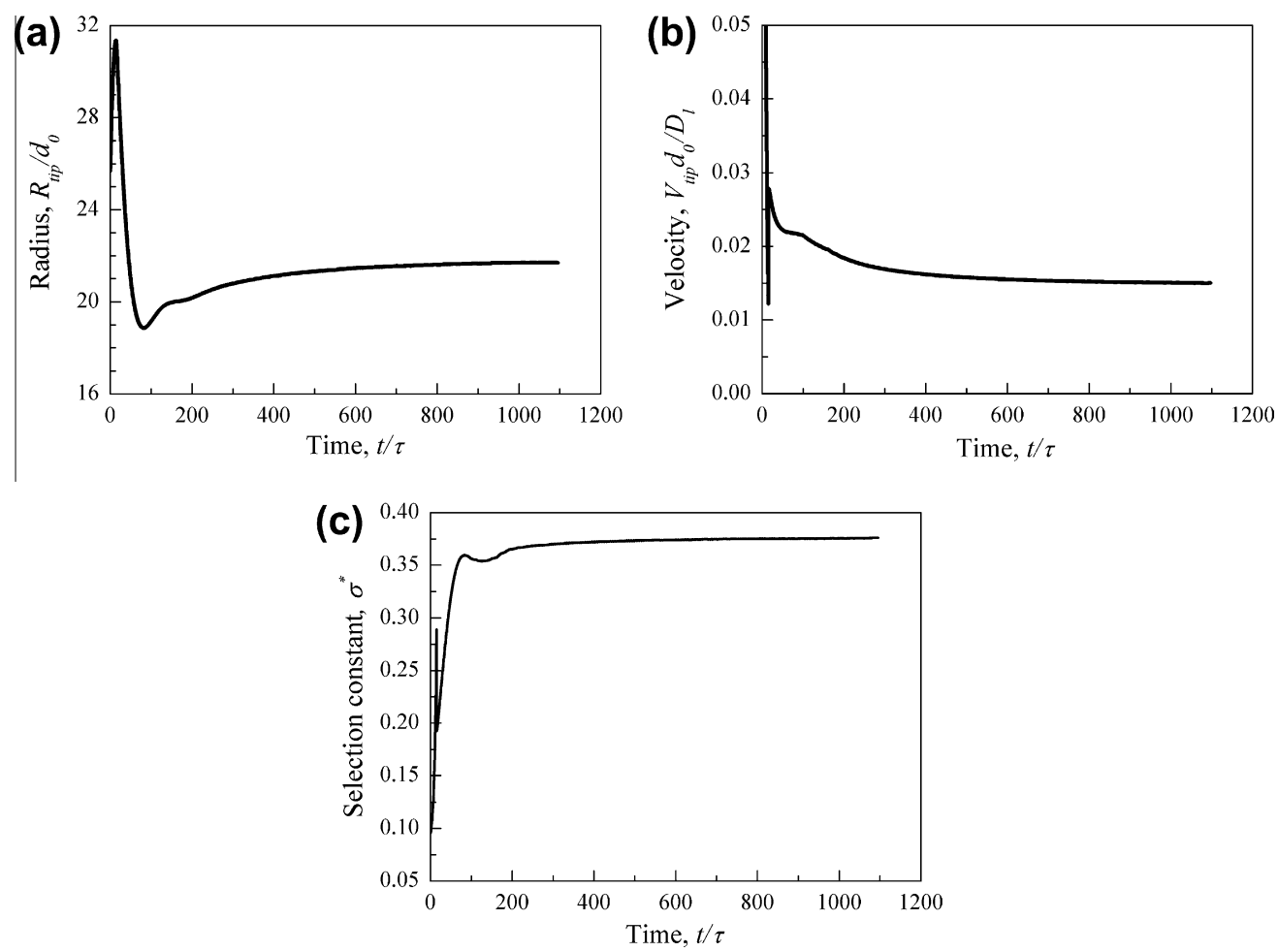

Fig. 2. Evolution of (a) tip radius, (b) tip velocity and (c) selection constant with time. The supersaturation is 0.55 and the interface width is $5 d_{0}$ in the simulation. 
and $V_{\text {tip. }}$. The result is shown in Fig. 2c, where we see that (as is commonly observed) $\sigma^{*}$ reaches a constant value well before the tip velocity and radius reach their steady state values. We use the steady-state tip radius and tip velocity to examine the convergence behaviour as a function of the simulation parameters.

\section{Using interface Peclet number to determine the maximum simulation interface width}

\subsection{Issues with the $w / d_{0}$ criterion to find the $w_{\max }$}

Convergence analysis is done to assess the calculation accuracy by examining (1) concentration profile, (2) tip radius and (3) tip growth velocity. The solute concentration in the solid part at the point $x=800 d_{0}$ of the dendrite growing at the supersaturation of 0.55 was calculated using different simulation interface width $(w)$ varying from $1.8 d_{0}$ to $10 d_{0} . x=800 d_{0}$ is about the middle point of the solidified steady-state dendrite. The calculated lowest concentration is $0.139 C_{l}^{0}$ when $w$ is as small as $3.5 d_{0}$, the calculated largest concentration is $0.147 C_{l}^{0}$ at the largest $w$, and accordingly fluctuation is within $6 \%$. Therefore, the convergence analysis in solute concentration reveals that the result of solute concentration is not sensitive to the simulation interface width in the range from $1.8 d_{0}$ to $10 d_{0}$. This is attributed to the inclusion of the anti-trapping term in the solute diffusion equation of Eq. (4) $[8,9]$.

The calculated steady-state dendrite tip radius $\left(R_{\text {tip }}\right)$ vs. simulation interface width $(w)$ is shown in Fig. 3 (solid line with square symbols) for $\Omega=0.55$. The results converge well when the interface width is smaller than $6.25 d_{0}$ with a convergent value around $21 d_{0}$ and the fluctuation in this range is less than $5 \%$. A sudden increase can be observed at $w=7.2 d_{0}$ of $R_{\text {tip }}=25 d_{0}$ with a $19 \%$ variation. The calculated tip radius continues to increase with $w$ increasing and reaches as high as $36 d_{0}$ at $w=10 d_{0}$. The plot of steady-state tip velocities $\left(V_{\text {tip }}\right)$ using different $w$ is also shown in Fig. 3 as solid line with sphere symbols. The convergent value is about $0.015 D_{l} / d_{0}$ when $w \leq 5 d_{0}$ and the fluctuation in this zone is less $2 \%$. The tip velocity suddenly increases to $0.018 D_{l} / d_{0}$ with a $20 \%$ variation at $w=5.5 d_{0}$ and then decreases with a sharp slope when $w$ increases.

Based on the results described above, it can be observed in Fig. 3 that $w$ limit of convergence in tip velocity is smaller than that in tip radius. At $\Omega=0.55$, the tip velocity at $w=5.5 d_{0}$ deviates from the converged value by about $20 \%$ increase, while the calculated tip radius remains accurate. The Ivantsov transport solution suggests that $R_{\text {tip }} V_{\text {tip }}$ should be roughly constant [37], but the solution obtained by phase-field simulation at $w=5.5 d_{0}$ deviates significantly. Furthermore, the increase in calculated tip velocity means the decrease of $D_{l} / V_{p}$ which is due to the use of the larger $w$. Interface

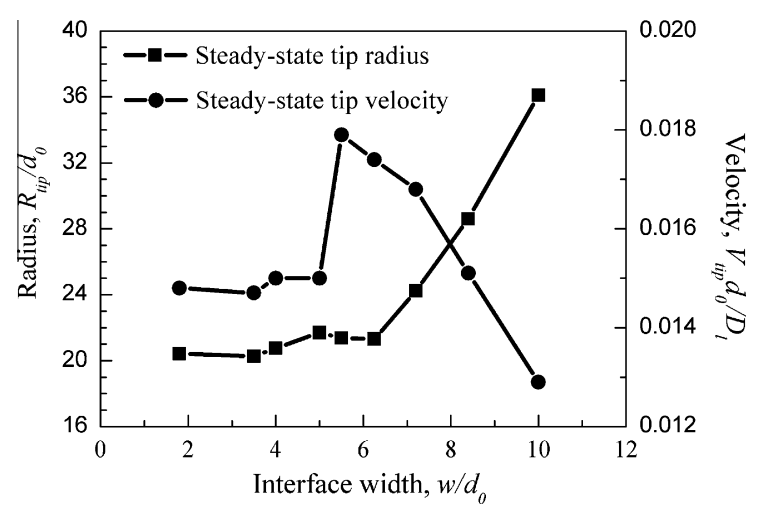

Fig. 3. Calculated steady-state tip radius and tip velocity at different interface width.

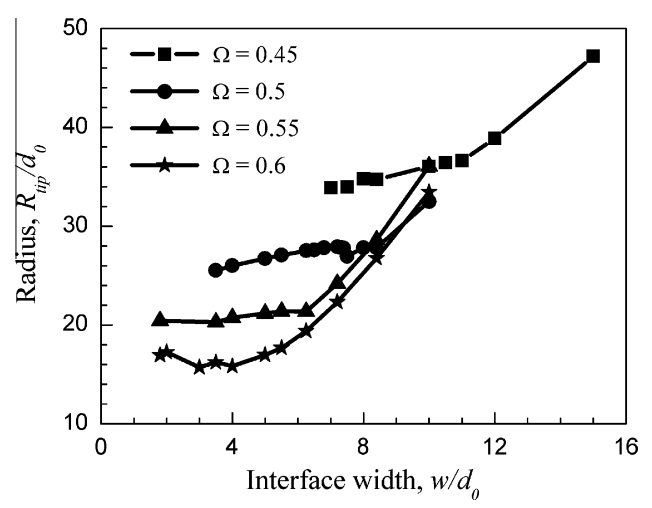

Fig. 4. Calculated $R_{\text {tip }}$ vs. $w$ at different supersaturations $\Omega$.

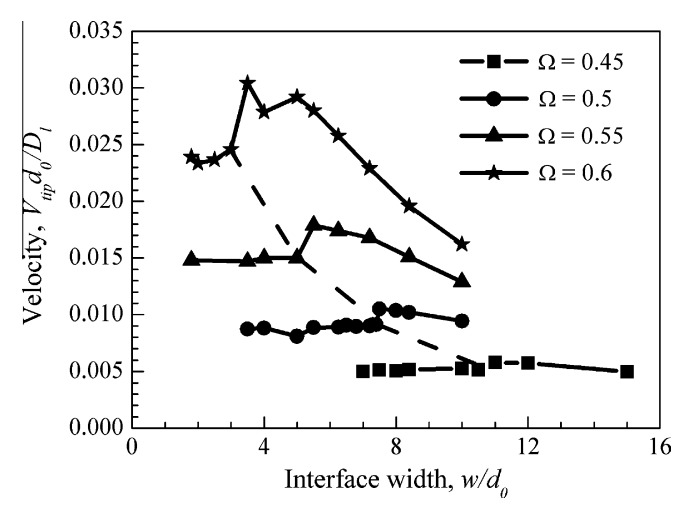

Fig. 5. Calculated $V_{\text {tip }}$ vs. $w$ at different supersaturations $\Omega$.

Peclet number is expressed as $P=w /\left(D_{l} / V_{\text {tip }}\right)$ and therefore a sudden jump would be observed at $w=5.5 d_{0}$ corresponding to the jump in velocity, which will be discussed further below.

Fig. 4 shows the calculated $R_{\text {tip }}$ vs. $w$ at different supersaturations. For each $\Omega$, the obtained $R_{\text {tip }}$ converge well at small interface widths and then increase sharply. The convergence limit of $w$ is $12 d_{0}$ at $\Omega=0.45,8.4 d_{0}$ at $\Omega=0.5,6.25 d_{0}$ at $\Omega=0.55$ and then decreased to $4.0 d_{0}$ at $\Omega=0.6$. Fig. 5 shows the plots of $V_{\text {tip }}$ vs. $w$ at different supersaturations. The obtained growth velocity increases with higher supersaturation $\Omega$; the tip velocity at $\Omega=0.6$ is about 5 times as that at $\Omega=0.45$. Under a certain $\Omega$, the obtained steady-state tip velocity converges well at small interface widths, then increases to a peak value which is obviously different from the convergent value, and decrease with the increase in $w$. The convergence limit is $10.5 d_{0}$ at $\Omega=0.45$, $7.2 d_{0}$ at $\Omega=0.5,5 d_{0}$ at $\Omega=0.55$, and decreases to $3.0 d_{0}$ at the highest $\Omega=0.6$.

The maximum interface width $\left(w_{\max }\right)$ is the limit of the convergence zones in tip radius and the tip velocity to simulate the dendrite growth accurately. By this measure, $w_{\max }=10.5 d_{0}$ at $\Omega=0.45$, $w_{\max }=7.2 d_{0}$; at $\Omega=0.5, w_{\max }=5 d_{0}$ at $\Omega=0.55$, and $w_{\max }=3.0 d_{0}$ at $\Omega=0.6$, which is shown as a dashed line in Fig. 5. It can be seen that after the limit, a sudden increase in tip velocity is observed for each $\Omega$. Fig. 5 illustrates that the maximum interface width increases with the growth velocity decreases. Then there needs a parameter that can limit $w_{\max }$ even under the relatively low growth velocity.

The growth velocity of the dendrite varies with solidification time. As shown by the above results, $w_{\max } / d_{0}$ decreases with the growth velocities increase, meaning that $w_{\max }$ should be selected according to the maximum growth velocity. It is impossible to use $w / d_{0}$ criterion to select the maximum interface width for the 
transient dendrite growth because $w / d_{0}$ criterion has no link to the growth velocity. By contrast, the interface Peclet number, $P=w /$ $\left(D_{l} / V_{\text {tip }}\right)$, is related to the growth velocity. Thus the question is: can a value be used to select maximum simulation width for transient dendrite growth using the interface Peclet number $P=w /\left(D_{l}\right)$ $\left.V_{\text {tip }}\right)$ ?

\subsection{Using interface Peclet number $P=w /\left(D_{l} / V_{\text {tip }}\right)$ to determine the $w_{\max }$}

Echebarria et al. [9] expressed the possibility to use the interface Peclet number, $P=w /\left(D_{l} / V_{t i p}\right)$, as a small expansion parameter in the thin-interface analysis. Because of the interface stretching and surface diffusion terms appearing at second order and third order in an expansion in the interface Peclet number, this choice was not derived mathematically. In this study, the feasibility of using interface Peclet number to select the interface simulation width will be analysed by examining numerical simulation results.

The calculated steady-state tip velocity as shown in Fig. 5 are plotted against the interface Peclet number $(P)$ in Fig. 6. It should be mentioned here that the larger $P$ in Fig. 6 corresponds to larger interface width $w$ in Fig. 5 under each supersaturation. The plot of $V_{\text {tip }}$ Vs. $P$ has the same trend of the plot of $V_{\text {tip }}$ Vs. $w$; the obtained tip velocity converges well at small $P$ and goes to a peak value outside the convergence zone. Again, a dash line was drawn as the boundary of the convergence zone. As shown in Fig. 6, the convergence limit of $P$ is around 0.075 for $\Omega=0.55$ and $\Omega=0.6$, corresponding to $w=5 d_{0}$ and $w=3 d_{0}$ respectively. At $\Omega=0.5$ the convergence limit of $P$ decreased to 0.067 and continuously decreased to 0.054 at $\Omega=0.45$. The results show that the $P$ limit slightly decreased with the growth velocity decreased, opposite to the $w / d_{0}$ criterion. Importantly, $P=0.075$ is its limit; when $P>0.075$, the simulation results become unreliable.

Compared to $w / d_{0}$, it makes more sense to use $P=w /\left(D_{l} / V_{\text {tip }}\right)$ as an expansion parameter because the limit of $P$ is comparable to zero with the value of 0.075 obtained by the above numerical results. When $P>0.075$, the solution dependent on the non-linear order on $P$ could not vanish and the PF simulation results only consider the linear order will largely deviate the accurate results. $P$ is proportional to the growth velocity at constant $w$ and $D_{l}$, indicating that the simulation interface width used should change significantly when the velocity changes greatly. This explains why the value of $w / d_{0}$ spans a large range in the literatures, as shown in Table 1 . To examine the selected interface width $w$ is within the $P$ limit, $P$ values were calculated for the cases in Table 1 and are shown in Table 2 plus the $P$ values in Ref. [1]. It could be seen that the $P$ values chosen around the limit we obtain in Fig. 6. Having the $P$ limit in mind, it is straightforward to select the maximum interface width for the transient dendrite growth; $w<w_{\max }=0.075 D_{l} /$

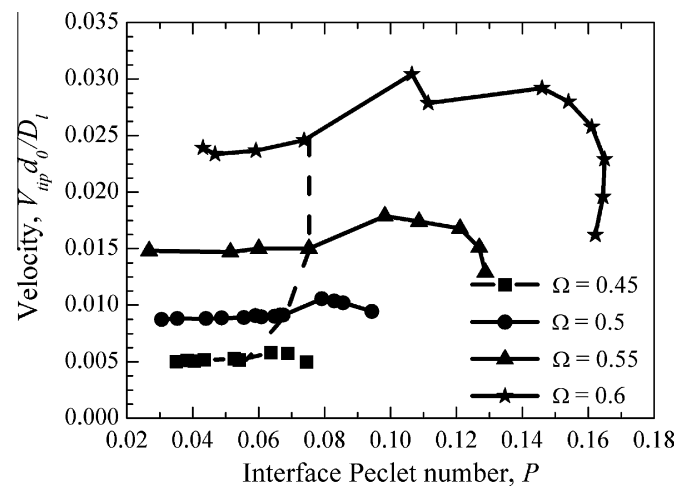

Fig. 6. Calculated $V_{\text {tip }}$ vs. $P$ at different supersaturations $\Omega$.
Table 2

Values of $P$ used in selecting interface width in phase-field simulations.

\begin{tabular}{|c|c|c|c|}
\hline$P$ & $w / d_{0}$ & Validation methods & Refs \\
\hline 0.027 & 1.83 & Compare with Gibbs-Thomson relation & {$[8]$} \\
\hline 0.051 & 3.6 & Compare with Gibbs-Thomson relation & [8] \\
\hline 0.030 & 72.1 & $\begin{array}{l}\text { Comparison with Mullins-Sekerka theory } \\
\text { Convergence analysis }\end{array}$ & [9] \\
\hline 0.040 & 36.1 & Comparison with experimental data & [1] \\
\hline 0.075 & 36.1 & Comparison with experimental data & [1] \\
\hline
\end{tabular}

$V_{\max }$ where $V_{\max }$ is the maximum growth velocity during transient dendrite growth.

\section{Effect of finite solid diffusivity on the $P$ limit in selecting the maximum interface width}

$P=w /\left(D_{l} / V_{\text {tip }}\right)$ does not include the expression of solid diffusivity $\left(D_{s}\right)$ and the effect of $D_{s}$ on the $P$ limit is important to select the maximum interface width for $\mathrm{Fe}-\mathrm{C}$ alloys. An extension of Karma's thin-interface model $[8,9]$ was proposed by Ohno and Matsuura (OM) [10] to include the solid diffusivity. In OM extension, Eqs. (7) and (9) are modified into:

$\tilde{q}(\phi)=\frac{(1-\phi)+k_{e} \frac{D_{s}}{D_{l}}(1+\phi)}{1+k_{e}-\left(1-k_{e}\right) \phi}$

$a(\phi)=\frac{[h(\phi)-1][1-q(\phi)]-\chi(s)\left[k_{e} \frac{D_{s}}{D_{l}}-q(\phi)\right][1-q(\phi)]}{\sqrt{2}\left(\phi^{2}-1\right)}$

with the solute flux term in solid $\chi(s)=2\left(\left.\partial_{\eta} U_{1}\right|^{-\infty}\right) / v_{n}$, where $\left(\left.\partial_{\eta} U_{1}\right|^{-\infty}\right)$ is the solute flux going into the solid and $v_{n}$ is the growth velocity normal to the interface. One problem with this model is that prior knowledge of $\chi(s)$ is needed to use as input in simulation. However $\chi(s)$ is an output of the simulation. In the numerical tests by OM, $\chi(s)$ was assumed to be zero $[10,25]$. When this assumption is made, two issues arise: (1) can one still recover the Gibbs-Thomson relation with a thin-interface width? and (2) will this change the $P$ limit in selecting the interface width? To examine these issues, isothermal simulations were carried out with different $D_{s} / D_{l}$, including $D_{s} / D_{l}=0.001$ of the same order of Al-Cu alloys, $D_{s} /$ $D_{l}=0.1$ of the same order of Fe-C alloys and $D_{s} / D_{l}=1$ of the symmetry in solute diffusivity in the solid and liquid.

Fig. 7 shows the dendrite tip concentration predicted by $\mathrm{OM}$ extension and by Gibbs-Thomson relation using the interface Peclet number $P=0.055$ at $D_{s} / D_{l}=0.1$. The evolution of $C_{\text {tip }}$ calculated by the Gibbs-Thomson relation is shown in dash line; the tip radius $R_{t i p}$ was obtained by PF simulation where the interface width

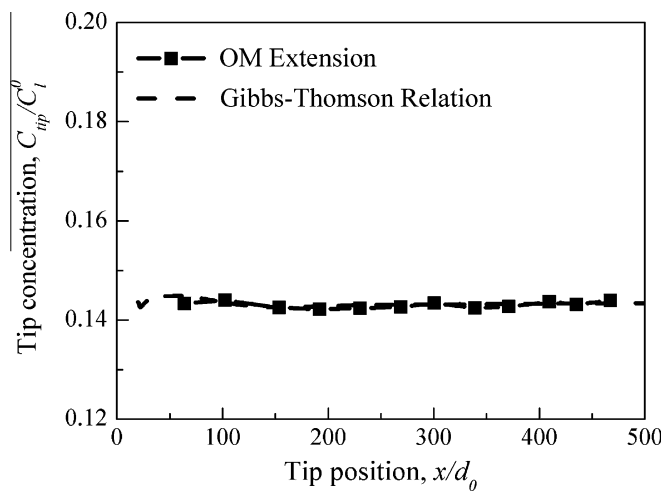

Fig. 7. The dendrite tip concentration predicted by $\mathrm{OM}$ extension with $P=0.055$ and by Gibbs-Thomson relation. 


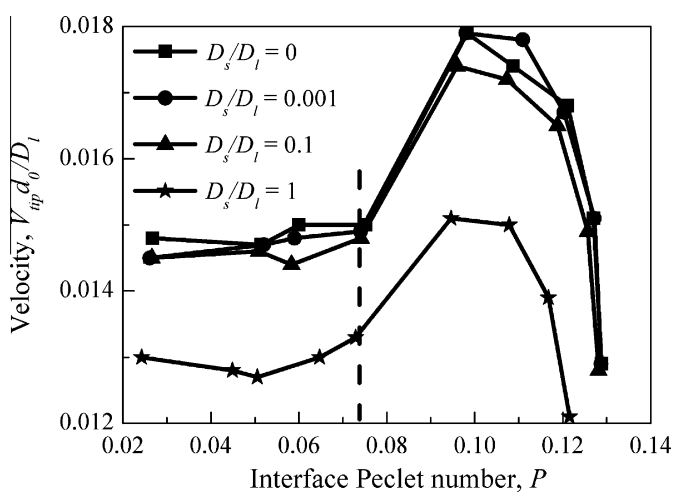

Fig. 8. Calculated $V_{\text {tip }}$ vs. $P$ at different solid diffusivities $D_{s} / D_{l}$.

$w=4 d_{0}$ was used. The tip concentration calculated by the $\mathrm{OM}$ extension is shown as black solid line; the tip position and tip concentration were obtained at times $t=50 \tau_{0}-600 \tau_{0}$ with the time interval $\Delta t=50 \tau_{0}$. It can be seen that the predicted dendrite tip concentration agrees well with that obtained by Gibbs-Thomson relation of $2 \%$ disagreement, indicating that this model can recover the Gibbs-Thomson relation using a thin-interface width of $P=0.055$ although the assumption $\chi(s)=0$.

Convergence analysis for different $D_{s} / D_{l}$, including $0,0.001,0.1$ and 1.0 , were subsequently performed. Fig. 8 plots $V_{\text {tip }}$ vs. $P$ for different $D_{s} / D_{l}$. The convergence analysis in tip growth velocity is presented here rather than that in solute concentration and the tip radius because the calculated growth velocity goes beyond the convergent value first among these three quantities. As shown in Fig. 8, under the isothermal solidification with the same supersaturation, the growth velocity decreases with the $D_{s} / D_{l}$ increasing. Furthermore, the degree of the decrease is not obvious even $D_{s} / D_{l}$ is as large as 0.1 and obvious decrease could be observed at $D_{s} /$ $D_{l}=1.0$. A line was drawn to distinguish the convergence zone. It can be seen that this line is almost vertical to the $x$-axis, indicating that the $P$ limit at non-zero $D_{s} / D_{l}$ is the same as that at zero $D_{s} / D_{l}$. It indicates that the consideration in solid diffusivity has little influence on the $P$ limit to select the maximum interface width.

In our simulations, we assumed that $\chi(s)=0$ even for non-zero $D_{s} / D_{l}$. If the dependence of $\chi(s)$ does not vanish as the interface tends to be zero, setting $\chi(s)=0$ will lead to an inaccurate result even when a nano-scale interface width is set. We have carried out a mathematical derivation that shows that the terms related to $\chi(s)$ does vanish, which will be presented in another paper. Here, we examined the convergence behaviour of the model with finite $D_{s} / D_{l}$. The result is presented in Fig. 8, where we see that the upper limit for $\mathrm{P}$ is the same as for $D_{s}=0$.

The results shown above give a limit of the interface Peclet number $P$ within the convergence zone at varied growth velocities. Simulation results within the convergence zone can be obtained only when $P \leq 0.075$. The inclusion of the solute diffusion in solid has little influence on the limit value of $P$. It indicated that when choosing the simulation interface width for simulating dendritic growth under transient growth conditions, the maximum interface width needs to be selected based on the highest growth velocity to obtain accurate results.

\section{Conclusions}

This study explores the use of interface Peclet number $(P)$, $P=w /\left(D_{l} / V_{\text {tip }}\right)$, to determine the maximum simulation interface width for a thin-interface phase-field model. It was found that simulation results within the convergence zone can be obtained only when $P<0.075$. This reveals that a maximum interface width for the transient dendrite growth is $w_{\max }<0.075 D_{l} / V_{\max }$ where $V_{\max }$ is the maximum growth velocity. The inclusion of the solute diffusion in solid has little influence on the limit value of $P$.

\section{Acknowledgements}

This research work is supported by the European Commission as part of the FP7 programme, as the project, Modelling of Interface Evolution in Advanced Welding; Contract No. NMP3-SL-2009229108.

\section{References}

[1] B.P. Athreya, J.A. Dantzig, S. Liu, R. Trivedi, Philos. Mag. 86 (2006) 3739

[2] I. Steinbach, Acta Mater. 56 (2008) 4965.

[3] W.J. Boettinger, J.A. Warren, C. Beckermann, A. Karma, Annu. Rev. Mater. Res. 32 (2002) 163.

[4] A.A. Wheeler, W.J. Boettinger, G.B. McFadden, Phys. Rev. A 45 (1992) 7424.

[5] S.G. Kim, W.T. Kim, T. Suzuki, Phys. Rev. E 58 (1998) 3316.

[6] A. Karma, Phys. Rev. E 60 (1999) 3614.

[7] S.G. Kim, W.T. Kim, T. Suzuki, Phys. Rev. E 60 (1999) 7186.

[8] A. Karma, Phys. Rev. Lett. 87 (2001) 115701.

[9] B. Echebarria, R. Folch, A. Karma, M. Plapp, Phys. Rev. E 70 (2004) 061604.

[10] M. Ohno, K. Matsuura, Phys. Rev. E 79 (2009) 031603.

[11] M. Asta, C. Beckermann, A. Karma, W. Kurz, R. Napolitano, M. Plapp, G. Purdy, M. Rappaz, R. Trivedi, Acta Mater. 57 (2009) 941.

[12] Y. Chen, Acta Mater. 60 (2012) 199.

[13] P.R. Cha, D.H. Yeon, J.K. Yoon, Acta Mater. 49 (2001) 3295.

[14] B. Böttger, J. Eiken, M. Ohno, G. Klaus, M. Fehlbier, R. Schmid-Fetzer, I Steinbach, A. Bührig-Polaczek, Adv. Eng. Mater. 8 (2006) 241.

[15] B. Böttger, J. Eiken, I. Steinbach, Acta Mater. 54 (2006) 2697.

[16] S.G. Kim, Acta Mater. 55 (2007) 4391.

[17] B. Böttger, M. Apel, J. Eiken, P. Schaffnit, I. Steinbach, Steel Res. Int. 79 (2008) 608.

[18] S.G. Fries, B. Boettger, J. Eiken, I. Steinbach, Int. J. Mater. Res. 100 (2009) 128

[19] P.K. Galenko, S. Reutzel, D.M. Herlach, S.G. Fries, I. Steinbach, M. Apel, Acta Mater. 57 (2009) 6166.

[20] R. Folch, M. Plapp, Phys. Rev. E 72 (2005) 011602.

[21] J. Eiken, B. Böttger, I. Steinbach, Phys. Rev. E 73 (2006) 066122.

[22] I. Steinbach, M. Apel, Physica D 217 (2006) 153.

[23] I. Steinbach, B. Böttger, J. Eiken, N. Warnken, S.G. Fries, J. Phase Equilib. Diff. 28 (2007) 101.

[24] I. Steinbach, Model. Simul. Mater. Sci. Eng. 17 (2009) 073001.

[25] M. Ohno, K. Matsuura, Acta Mater. 58 (2010) 5749.

[26] N. Warnken, D. Ma, A. Drevermann, R.C. Reed, S.G. Fries, I. Steinbach, Acta Mater. 57 (2009) 5862.

[27] J.Q. Broughton, G.H. Gilmer, K.A. Jackson, Phys. Rev. Lett. 49 (1982) 1496.

[28] L.V. Mikheev, A.A. Chernov, J. Cryst. Growth 112 (1991) 591.

[29] A. Karma A, W-J Rappel, Phys. Rev. E 53 (1996) R3017.

[30] A. Karma, J. Cryst. Growth 174 (1997) 54.

[31] A. Karma, Phys. Rev. E 57 (1998) 4323.

[32] S. Gurevich, M. Amoorezaei, N. Provatas, Phys. Rev. E 82 (2010) 051606.

[33] M. Amoorezaei, S. Gurevich, N. Provatas, Acta Mater. 58 (2010) 6115.

[34] V. Fallah, M. Amoorezaei, N. Provatas, S.F. Corbin, A. Khajepour, Acta Mater. 60 (2012) 1633.

[35] J.-H. Jeong, Phys. Rev. E 64 (2001) 041602.

[36] N. Provatas, M. Greenwood, B. Athreya, N. Goldenfeld, J.A. Dantzig, Int. J. Mod. Phys. B 19 (2005) 4525

[37] J.A. Dantzig, M. Rappaz, Solidification, first ed., EPFL Press, Switzerland, 2009. 